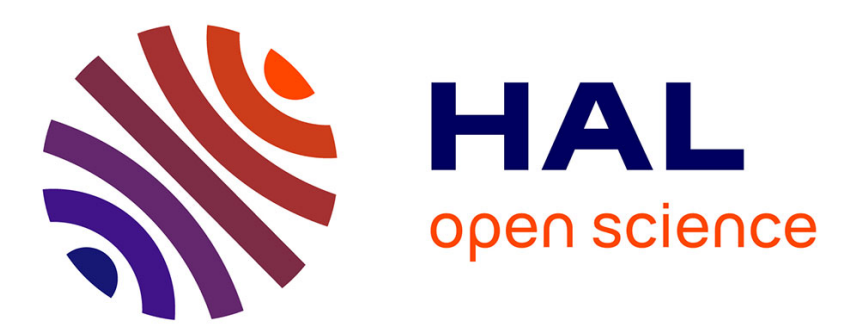

\title{
On the Model Order Reduction of Confined Plasticity
}

Mohamed Aziz Nasri, Amine Ammar, Francisco Chinesta, Camille Robert, Saber El Arem, Franck Morel

\section{To cite this version:}

Mohamed Aziz Nasri, Amine Ammar, Francisco Chinesta, Camille Robert, Saber El Arem, et al.. On the Model Order Reduction of Confined Plasticity. ESAFORM (19; 2016; Nantes), Apr 2016, Nantes, France. pp.6. hal-01310812

\section{HAL Id: hal-01310812 https://hal.science/hal-01310812}

Submitted on 3 May 2016

HAL is a multi-disciplinary open access archive for the deposit and dissemination of scientific research documents, whether they are published or not. The documents may come from teaching and research institutions in France or abroad, or from public or private research centers.
L'archive ouverte pluridisciplinaire HAL, est destinée au dépôt et à la diffusion de documents scientifiques de niveau recherche, publiés ou non, émanant des établissements d'enseignement et de recherche français ou étrangers, des laboratoires publics ou privés. 


\title{
On the Model Order Reduction of Confined Plasticity
}

\author{
Mohamed Aziz Nasri ${ }^{1, a), b)}$, Amine Ammar ${ }^{1,2, c)}$, Francisco Chinesta ${ }^{3, d)}$, Camille \\ Robert $^{1, \mathrm{e})}$, Saber ElArem ${ }^{1, \mathrm{f})}$ and Franck Morel ${ }^{1, \mathrm{~g})}$ \\ ${ }^{1}$ LAMPA, Arts et Métiers ParisTech at Angers \\ 2 Boulevard du Ronceray, BP 93525, F-49035 Angers cedex 01, France \\ ${ }^{2}$ UMSSDT, ENSIT, Université de Tunis \\ 5 Avenue Taha Hussien, Montfleury 1008, Tunis, Tunisie \\ ${ }^{3}$ GeM UMR CNRS-Centrale Nantes, 1 rue de la Noe, F-44300 Nantes, France.

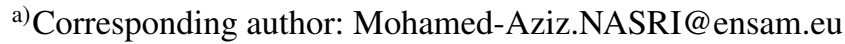 \\ b)Mohamed-Aziz.NASRI@ensam.eu \\ c)Amine.AMMAR@ensam.eu

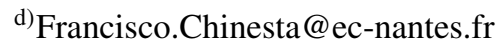 \\ e)Camille.Robert@ensam.eu \\ ${ }^{\text {f) }}$ Saber.Elarem@ensam.eu \\ ${ }^{\mathrm{g})}$ Franck.Morel@ensam.eu
}

\begin{abstract}
Forming processes usually involve irreversible plastic transformations. The calculation in that case becomes cumbersome when large parts and processes are considered. Recently Model Order Reduction techniques opened new perspectives for an accurate and fast simulation of mechanical systems. In some processes, plastic deformations remain very localized, for example in the immediate neighborhood of the surface. In that case, the in-plane characteristic dimension is several orders of magnitude higher than the one related to the deepness in which plasticity localizes. In those situations the use of standard mesh-based 3D discretization is challenging because the extremely different characteristic dimensions that to capture all the information requires the use of millions of nodes.
\end{abstract}

\section{INTRODUCTION}

In elastoplascitity, the behavior at each position and time depends on all the previous mechanical history as well as on the present loading. Obviously if the deformation history is given at each position, the elastoplastic behavior law can be easily integrated in order to compute the stress evolution. However such an information is not generally available when proceeding with standard discretization strategies where the solution is computed incrementally.

When using standard incremental discretization techniques we must proceed differently. We assume at time $t_{n}$ (time in the sense of loading) that the state of the systems is perfectly defined, verifying the equilibrium and the constitutive equations. The mechanical state is given by $\epsilon_{n}, \sigma_{n}, \epsilon_{n}^{p}$ and $\alpha_{n}$, the last being the internal variable describing the material hardening (accumulated plastic deformation, plastic work, ... ) assumed here isotropic without loss of generality.

The equilibrium at time $t_{n}$ writes in discrete form:

$$
\mathbf{F}^{i n t}\left(\sigma_{n}\right)=\mathbf{F}_{n}^{e x t}
$$

Now, an increment of load applies due to volume forces or surface tractions, and the problem consists in computing the displacement change $\Delta u_{n+1}$ and the associated final state defined by $\epsilon_{n+1}, \sigma_{n+1}, \epsilon_{n+1}^{p}$ and $\alpha_{n+1}$. The new state must verify both the equilibrium equation

$$
\mathbf{F}^{\text {int }}\left(\sigma_{n+1}\right)=\mathbf{F}_{n+1}^{e x t}
$$

and the rate-independent elastoplastic constitutive equations. As the problem is nonlinear we must iterate. 
General discretization techniques proceed by solving incrementally the balance and constitutive equations, within the implicit or explicit framework. The so-called non-incremental techniques iterate between the stress and displacement history-fields verifying the equilibrium and the constitutive equation. Space-time separated representations within the Proper Generalized Decomposition [1, 3, 5, 7] allow performing such a decoupled integration [10, 11].

In the present work we focus in a very different issue, the one related to confined elastoplasticity, as the one encountered when performing surface treatments by applying plastic deformations in a very thin layer at the immediate surface neighborhood. Because the micro-metric characteristic deepness of the plastic layer, and its large in-plane dimension a 3D simulation remains a challenging issue because the number of elements can become rapidly unaffordable.

In these circumstances an in-plane-out-of-plane separated representation allows circumventing the just referred issue, because the mesh in the thickness direction can be extremely fine without compromising the efficiency of the resulting integration procedure. This kind of decomposition has been widely considered in our former works, for solving a variety of physics in different degenerated domains, including plates, shells, rod-like geometries, and also laminates constituted by tens of plies along the thickness direction. The interested reader can refer to [2, 4, 6, 8, 9] and the references therein.

\section{INCREMENTAL ELASTOPLASTIC MODEL}

\section{Elastoplastic model}

The elastic behavior is given by

$$
\sigma=\mathbf{C} \epsilon
$$

The main steps of the calculation are:

- Compute the deviatoric stress $\sigma^{\prime}$ from

$$
\sigma^{\prime}=\sigma-(1+v) \operatorname{Tr}(\sigma)
$$

where $v$ is the Poisson coefficient.

- Determine the equivalent Von-Mises stress

$$
\sigma_{e}=\sqrt{\frac{3}{2} \sigma^{\prime}: \sigma^{\prime}}
$$

- Determine the yield function

$$
f=\sigma_{e}-\left(r+\sigma_{y}\right)
$$

where $r$ is the hardening and $\sigma_{y}$ the yield stress.

- Determine if yielding occurs, that is, if $f>0$. If $f \leq 0$ then the plastic multiplayer $d \lambda$ vanishes $d \lambda=0$. On the contrary, i.e. if $f>0$ the plastic multiplayer persistency condition results in

$$
d \lambda=\frac{\mathbf{n} \cdot C d \epsilon}{\mathbf{n} \cdot C \mathbf{n}+h}
$$

where $h$ is the plastic tangent modulus and $\mathbf{n}$ is given by

$$
\mathbf{n}=\frac{\partial f}{\partial \sigma}=\frac{3}{2} \frac{\sigma^{\prime}}{\sigma_{e}}
$$

- Determine the stress and the isotropic hardening increments:

$$
d \sigma=\mathbf{C} d \epsilon^{e}=\mathbf{C}(d \epsilon-d \lambda \mathbf{n})
$$

and

$$
d r=h d \lambda
$$


- Update all the quantities

$$
\left\{\begin{array}{l}
\sigma(\mathbf{x}, t+\Delta t)=\sigma(\mathbf{x}, t)+d \sigma \\
\epsilon^{p}(\mathbf{x}, t+\Delta t)=\epsilon^{p}(\mathbf{x}, t)+d \lambda \mathbf{n} \\
r(t+\Delta t)=r(t)+d r
\end{array}\right.
$$

The equilibrium writes (using vector notation)

$$
\int_{\Omega} \epsilon^{*} \cdot d \sigma d \mathbf{x}=\int_{\partial_{t} \Omega} \mathbf{u}^{*} \cdot d \mathbf{t} d \mathbf{x}
$$

or

$$
\int_{\Omega} \epsilon^{*} \cdot \mathbf{C} d \epsilon d \mathbf{x}=\int_{\Omega} \epsilon^{*} \cdot \mathbf{C} d \lambda \mathbf{n} d \mathbf{x}+\int_{\partial_{t} \Omega} \mathbf{u}^{*} \cdot d \mathbf{t} d \mathbf{x}
$$

where $d \mathbf{t}$ represents the load increment.

Now, a standard finite element discretization results in

$$
\int_{\Omega} \epsilon^{*} \cdot \mathbf{C} d \epsilon d \mathbf{x}=\mathbf{U}^{* T} \mathbf{K d} \mathbf{U}
$$

and

$$
\int_{\Omega} \epsilon^{*} \cdot \mathbf{C} d \lambda \mathbf{n} d \mathbf{x}+\int_{\partial_{t} \Omega} \mathbf{u}^{*} \cdot d \mathbf{t} d \mathbf{x}=\mathbf{U}^{* T} \mathbf{d} \mathbf{F}
$$

\section{Space-time separated representation within an incremental integration scheme}

If we consider $n$ time steps (again time in the sense of loading) we can write

$$
\left\{\begin{aligned}
\mathbf{K d U _ { 1 }} & =\mathbf{d F}_{1} \\
\mathbf{K d U _ { 2 }} & =\mathbf{d F}_{2} \\
\vdots & \\
\mathbf{K d U}_{n} & =\mathbf{d F}_{n}
\end{aligned}\right.
$$

Now, we can apply the singular value decomposition to the matrix $\mathcal{F}$

$$
\mathcal{F}=\left[\begin{array}{llll}
\mathbf{d F}_{1} & \mathbf{d F}_{2} & \cdots & \mathbf{d F}_{n}
\end{array}\right]
$$

that allows approximating it from

$$
\mathcal{F} \approx \sum_{i=1}^{N_{F}} \mathbf{R}_{i} \otimes \mathbf{S}_{i}
$$

With the unknown field $\mathbf{d} \mathbf{U}(t)$ expressed in the separated non-incremental form

$$
\mathbf{d U}=\sum_{j=1}^{N_{U}} \mathbf{X}_{j} \otimes \mathbf{T}_{j}
$$

and using tensor notation the problem becomes

$$
(\mathbf{K} \otimes \mathbf{I}) \sum_{j=1}^{N_{U}} \mathbf{X}_{j} \otimes \mathbf{T}_{j}=\sum_{i=1}^{N_{F}} \mathbf{R}_{i} \otimes \mathbf{S}_{i}
$$

The main drawback of such a procedure is the necessity of reconstructing the plastic history and then applying a SVD to the large resulting matrix to perform its space-time separation. To alleviate such a calculation in [12] we proposed the use of a POD-based reduced basis for the plastic history representation. 


\section{IN-PLANE-OUT-OF-PLANE SEPARATED REPRESENTATION}

The in-plane-out-of-plane separated representation allows for solution of full 3D models defined in plate geometries with a computational complexity characteristic of $2 \mathrm{D}$ simulations. This separated representation allows for independent representation of the in-plane and off-plane fields dependencies. The main idea lies in the separated representation of the displacement field by using functions depending on the in-plane coordinates $\mathbf{x}=(x, y), \mathbf{P}_{i}^{j}(x, y)$, and others depending on the thickness direction $z, \mathbf{T}_{i}^{j}(z), j=1,2,3$ and $i=1, \cdots, N$ according to:

$$
\mathbf{u}(\mathbf{x}, z)=\left(\begin{array}{c}
u(\mathbf{x}, z) \\
v(\mathbf{x}, z) \\
w(\mathbf{x}, z)
\end{array}\right) \approx\left(\begin{array}{c}
\sum_{i=1}^{N} P_{i}^{1}(\mathbf{x}) \cdot T_{i}^{1}(z) \\
\sum_{i=1}^{N} P_{i}^{2}(\mathbf{x}) \cdot T_{i}^{2}(z) \\
\sum_{i=1}^{N} P_{i}^{3}(\mathbf{x}) \cdot T_{i}^{3}(z)
\end{array}\right),
$$

which leads to a separated representation of the strain. By introducing the latter into the flow problem weak form, it allows the calculation of functions $P_{i}(x, y)$ by solving the corresponding $2 \mathrm{D}$ equations and functions $T_{i}(z)$ by solving the associated $1 \mathrm{D}$ equations, as described later.

Eq. (21) can be rewritten in the compact form

$$
\mathbf{u}(\mathbf{x}, z) \approx \sum_{i=1}^{N} \mathbf{P}_{i}(\mathbf{x}) \circ \mathbf{T}_{i}(z),
$$

where "o" denotes the entry-wise or Hadamard's product.

Using notation in (21), the displacement gradient $\nabla \mathbf{u}(\mathbf{x}, z)$ can be written as:

$$
\begin{aligned}
\nabla \mathbf{u}=\left(\begin{array}{ccc}
\frac{\partial u}{\partial x} & \frac{\partial u}{\partial y} & \frac{\partial u}{\partial z} \\
\frac{\partial v}{\partial x} & \frac{\partial v}{\partial y} & \frac{\partial v}{\partial z} \\
\frac{\partial w}{\partial x} & \frac{\partial w}{\partial y} & \frac{\partial w}{\partial z}
\end{array}\right) \approx & \sum_{i=1}^{N}\left(\begin{array}{ccc}
\frac{\partial P_{i}^{1}}{\partial x} & \frac{\partial P_{i}^{1}}{\partial y} & P_{i}^{1} \\
\frac{\partial P_{i}^{2}}{\partial x} & \frac{\partial P_{i}^{2}}{\partial y} & P_{i}^{2} \\
\frac{\partial P_{i}^{3}}{\partial x} & \frac{\partial P_{i}^{3}}{\partial y} & P_{i}^{3}
\end{array}\right) \circ\left(\begin{array}{ccc}
T_{i}^{1} & T_{i}^{1} & \frac{\partial T_{i}^{1}}{\partial z} \\
T_{i}^{2} & T_{i}^{2} & \frac{\partial T_{i}^{2}}{\partial z} \\
T_{i}^{3} & T_{i}^{3} & \frac{\partial T_{i}^{3}}{\partial z}
\end{array}\right)= \\
& \sum_{i=1}^{N} \mathbb{P}_{i}(\mathbf{x}) \circ \mathbb{T}_{i}(z) .
\end{aligned}
$$

\section{NUMERICAL RESULTS}

For illustrating the potentialities of the incremental solution procedure making use of the in-plane-out-of-plane separated representation we consider in the present work a simple 2D rectangular domain very elongated $\Omega=$ $[-L, L] \times[0, H]$, with $L=1$ and $H=0.4$, by separating the horizontal and vertical coordinates within the framework just described, that consists in solving a sequence of $1 \mathrm{D}$ problems instead the standard 2D ones. A moving load is applied on the top boundary that moves to right and comes back to left during a given number of cycles. As the elastoplasticity is time-independent, the time must be associated with the load evolution. Fig. 1 illustrates the loading history. The material parameters are: $v=0.3, h / E=0.01$ and $\sigma_{y} / E=0.005$.

Fig. 2 depicts the in-plane modes (those concerning the $x$-coordinate) and the ones concerning the thickness direction for one of the first locations of the moving load. Finally Fig. 3 reconstructs the 2D displacement field on a mesh that consists of $200 \times 100$ nodes.

\section{REFERENCES}

[1] A. Ammar, B. Mokdad, F. Chinesta and R. Keunings, A new family of solvers for some classes of multidimensional partial differential equations encountered in kinetic theory modeling of complex fluids, J. NonNewtonian Fluid Mech., 139, 153-176, 2006. 


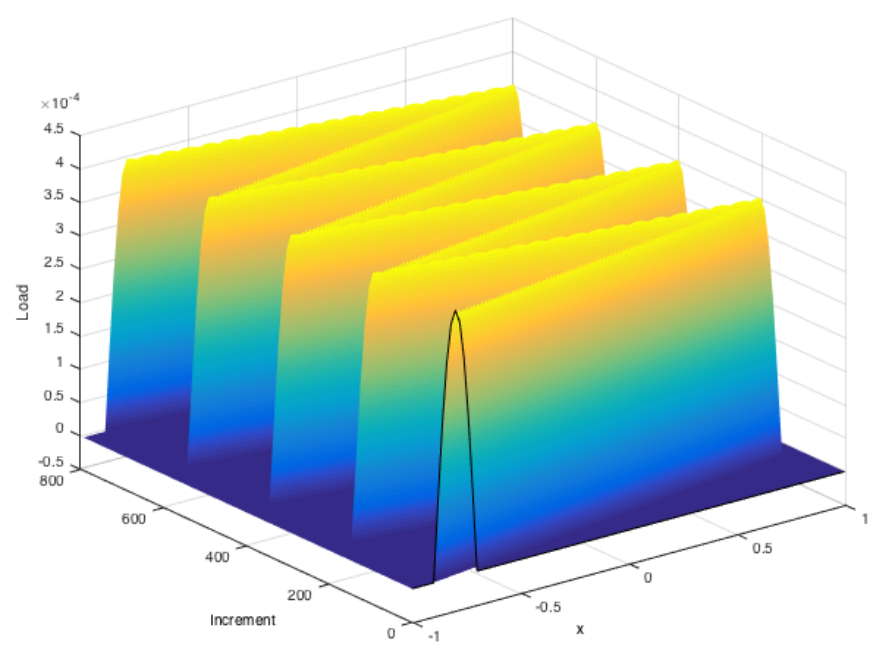

FIGURE 1. Applied cyclic loading acting on the top boundary.

[2] B. Bognet, A. Leygue, F. Chinesta, A. Poitou and F. Bordeu, Advanced simulation of models defined in plate geometries: 3D solutions with 2D computational complexity, Computer Methods in Applied Mechanics and Engineering, 201, 1-12, 2012.

[3] F. Chinesta, A. Ammar, E. Cueto. Recent advances and new challenges in the use of the Proper Generalized Decomposition for solving multidimensional models. Archives of Computational Methods in Engineering, 17/4, 327-350, 2010b.

[4] F. Chinesta, A. Ammar, A. Leygue and R. Keunings, An overview of the Proper Generalized Decomposition with applications in computational rheology, J. Non-Newtonian Fluid Mech., 166, 578-592, 2011.

[5] F. Chinesta, P. Ladeveze, E. Cueto. A short review in model order reduction based on Proper Generalized Decomposition. Archives of Computational Methods in Engineering, 18, 395-404, 2011.

[6] F. Chinesta, A. Leygue, B. Bognet, Ch. Ghnatios, F. Poulhaon, F. Bordeu, A. Barasinski, A. Poitou, S. Chatel and S. Maison-Le-Poec, First steps towards an advanced simulation of composites manufacturing by automated tape placement, International Journal of Material Forming, http://www.springerlink.com/index/10.1007/s12289-012-1112-9

[7] F. Chinesta, R. Keunings, A. Leygue. The Proper Generalized Decomposition for advanced numerical simulations. A primer. Springerbriefs, Springer, 2013.

[8] Ch. Ghnatios, F. Chinesta, Ch. Binetruy. The Squeeze Flow of Composite Laminates. International Journal of Material Forming, 8, 73-83, 2015.

[9] Ch. Ghnatios, E. Abisset-Chavanne, Ch. Binetruy, F. Chinesta, S. Advani. 3D Modeling of Squeeze Flow of Multiaxial Laminates. Journal of Non-Newtonian Fluid Mechanics.

[10] P. Ladeveze. On a family of algorithms for structural mechanics (in french). Comptes Rendus Académie des Sciences Paris, 300/2, 41-44, 1985.

[11] P. Ladeveze, The large time increment method for the analyze of structures with nonlinear constitutive relation described by internal variables, Comptes Rendus Académie des Sciences Paris, 309, 1095-1099, 1989.

[12] M.A. Nasri, J.V. Aguado, A. Ammar, E. Cueto, F. Chinesta, F. Morel, C. Robert, S. Elarem. Separated representation of incremental elastoplastic simulations Key Engineering Materials, 651-653, 1285-1293, 2015. 

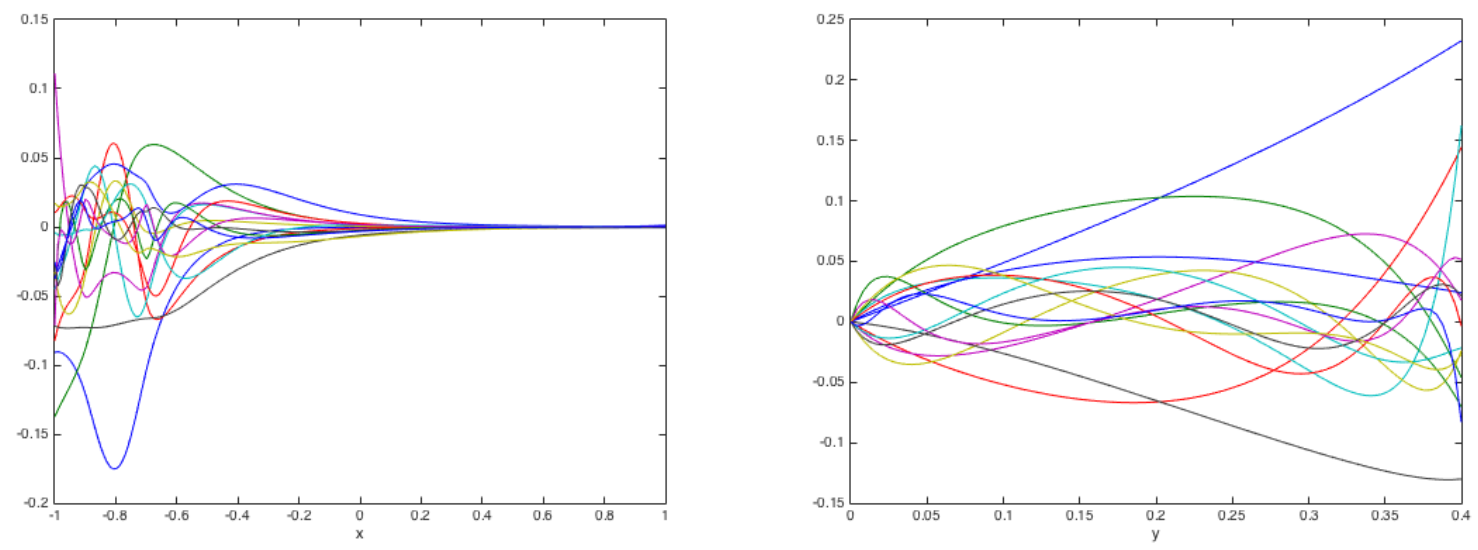

FIGURE 2. Main modes involved in the in-plane-out-of-plane separated representation: in-plane (left); out-of-plane (right), when load applies close to the left border.
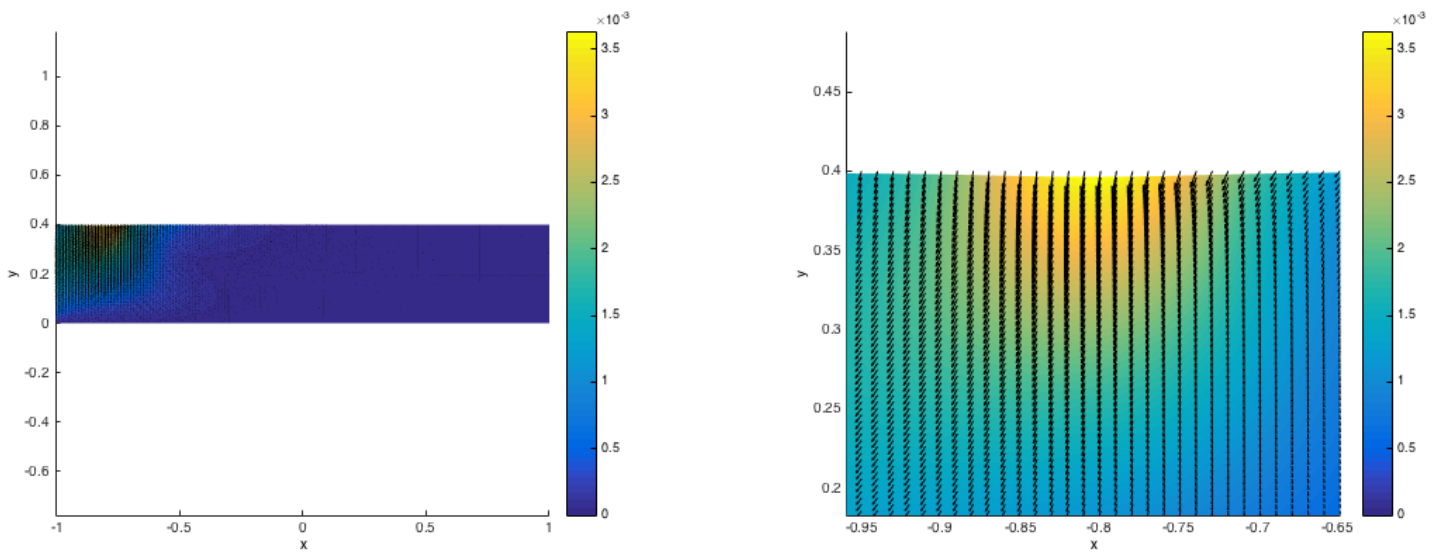

FIGURE 3. Reconstructuted 2D solution (left) and solution zoom (right). 